
\title{
25 Research Soure \\ Anti-VEGF Maintains The Function of Retinal Pigment Epithelium In Patients With Retinal Vein Occlusion
}

\section{Qian Yang}

Tongji University School of Medicine

Dawei Luo

Shanghai Jiao Tong University

Qinghua Qiu

Shanghai Jiao Tong University

Guo-Tong Xu ( $\nabla$ gtxu@tongji.edu.cn )

Tongji University School of Medicine https://orcid.org/0000-0002-0541-7214

Jingfa Zhang

Shanghai Jiao Tong University https://orcid.org/0000-0003-0601-4342

\section{Research Article}

Keywords: Anti-VEGF, retinal vein occlusion, macular edema, subretinal fluid, hyperreflective foci, RPE

Posted Date: January 31st, 2022

DOI: https://doi.org/10.21203/rs.3.rs-1245136/v1

License: (c) (i) This work is licensed under a Creative Commons Attribution 4.0 International License. Read Full License 


\section{Abstract}

Background: To evaluate the drainage function of retinal pigment epithelium (RPE) in patients with macular edema secondary to retinal vein occlusion (RVO-ME) after anti-vascular endothelial growth factor (anti-VEGF) treatment by optical coherence tomography angiography (OCTA).

Patients and Methods: Twenty-eight eyes with RVO-ME were imaged. Best-corrected visual acuity (BCVA), the area of the foveal avascular zone (FAZ), the nonperfusion areas (NPAs), the central macular thickness (CMT), the vascular density of the superficial/deep capillary plexus (SCP/DCP), the height of subretinal fluid (SRF), and the number of hyperreflective foci (HRF) were compared before and after treatments.

Results: Compared with pre-treatment, the CMT, NPAs, number of HRF and the height of SRF were decreased significantly after anti-VEGF treatment. There was no difference for BCVA, vascular density and FAZ area.

Conclusion: The absorption of SRF and the decrease of HRF indicated the restoration of RPE drainage function and anti-inflammation by anti-VEGF treatment.

\section{Background}

Retinal vein occlusion (RVO) is a relatively common retinal vascular disease which caused by the obstruction in central or branch retinal venous system, which is characterized by tortuous and dilatation of the retinal veins [1]. Clinically, RVO is divided into central retinal vein occlusion (CRVO) and branch retinal vein occlusion (BRVO) by the occlusion site. Hemiretinal vein occlusion (HRVO), also considered by some ophthalmologists to be a subgroup of CRVO, is an occlusion that causes about half of the retina to be affected [2]. According to statistics, the prevalence of BRVO is higher than CRVO, but the degree of visual impairment in patients with CRVO is more severer than that of BRVO [2].

Macular edema (ME) is the accumulation of the intraretinal or subretinal fluid in the macular region, and is one of the most common complications of RVO (RVO-ME) [3]. Although the pathogenesis of RVO-ME is not yet fully understood, the most accepted hypothesis is that venous compression leads to blood flow disorders, endothelial damage and thrombosis, and inflammation especially vascular endothelial growth factor (VEGF), is crucial in this process [4]. Based on the role of VEGF in the pathogenesis of RVO, antiVEGF has become an effective treatment and first-line therapy for the treatment of RVO-ME [2].

The destruction of the blood-retinal barrier (BRB) combined with the subsequent increased vascular permeability usually leads to $\mathrm{ME}$, which results in the visual impairment $[3,5]$. BRB is composed with inner BRB (iBRB, mainly consists endothelial cells and pericytes) and outer BRB (oBRB). The oBRB consists retinal pigment epithelium (RPE), and their tight junctions among RPE form the molecular components of oBRB [6]. Besides barrier function, RPE also drainage the subretinal fluid to the choroids to maintain relatively "dry" environment of subretinal space [7] through active ionic, aquaporins and other factors [8]. 
Optical coherence tomography angiography (OCTA) is widely used technique during the clinical practice due to the rapid and non-invasive imaging in patients [9]. With the help of OCTA, the vascular density in both SCP and DCP, NPA and hyperreflective foci (HRF) could be well defined in fundus diseases, including diabetic macular edema [10], RVO [11] and neovascular age-related macular degeneration [12].

In this study, we aimed to evaluate the drainage function of RPE before and after anti-VEGF treatment in patients with RVO-ME with the aid of OCTA. Besides the improvement in visual acuity and ME reduction, the SRF reduction after anti-VEGF indicated the improvement of drainage function of RPE by anti-VEGF treatment.

\section{Methods}

\section{Patients}

Twenty-eight consecutive treatment-naïve eyes from 28 RVO-ME patients who were diagnosed with comprehensive ophthalmologic examinations, between March 16, 2020, and July 1, 2021. The exclusion criteria were eyes with other diseases, such as diabetic retinopathy, hypertensive retinopathy, age-related macular degeneration, uveitis and other ocular diseases. In this retrospective study, the participants with two consecutive monthly intravitreal injections of anti-VEGF agents were enrolled.

All participants underwent full ophthalmologic examinations, including best-corrected visual acuity (BCVA), intraocular pressure (IOP) measurement, and anterior segment evaluation using slit-lamp biomicroscope, fundus photography, and OCTA imaging at the baseline and 1 week after treatment.

All intravitreal injections were performed by one experienced ophthalmologist. The intravitreal injection of anti-VEGF reagents (ranibizumab, Novartis Pharma Stein AG, Switzerland, $0.5 \mathrm{mg} / 0.05 \mathrm{~mL}$ or conbercept, Chengdu Kang Hong Biotech Co, Ltd, Sichuan, China, $0.5 \mathrm{mg} / 0.05 \mathrm{~mL}$ ) was conducted at the infratemporal quadrant through the eyeball's pars plana under aseptic conditions. About 1-week variation was allowed for every injection interval.

\section{Evaluation of Optical Coherence Tomography Angiography (OCTA).}

The examination with OCTA was refered to a published method [12]. Retinal microvasculature was imaged using the RTVue XR Avanti OCT system (Optovue, Inc., Fremont, CA, USA), and measurements were acquired using the manufacturer's AngioVue software.

The NPA were outlined manually in the enface image of the SCP and analyzed automatically by the OCTA auto-segmentation software. The number of HRF was manually counted within a $6 \mathrm{~mm}$ diameter centered on the foveal area using a fovea spanning horizontal B-scan. Measured the longest vertical distance between the highest point of the subretinal fluid and the interdigitation zone (IZ) / retinal pigment epithelial (RPE) layer as the height of the SRF. Poor quality images with a signal strength index< 
4/10 were excluded from further analysis. The quantification of HRF was carried out by two experienced physicians independently.

\section{Statistical Analyses.}

The data were analyzed by using GraphPad Prism 6.0 software. All values are presented as a number or mean \pm standard deviation. The visual acuity was presented as the logarithm of the minimum angle of resolution (logMAR). A paired t-test was used to compare BCVA, the vascular density of SCP and DCP, the FAZ area, NPA, the number of HRF and the height of SRF between pre-treatment and after 2 consecutive monthly anti-VEGF injections. A P value $<0.05$ was considered as statistical significance.

\section{Results}

\section{Demographics and clinical characteristics of the patients}

Twenty-eight eyes from 28 patients (12 males and 16 females) were enrolled in this study, as shown in Table 1. The mean age of patients was $64.18 \pm 10.97$ years old, ranging from 37 to 88 years old. The RVO includes 15 CRVO and 13 BRVO. All patients underwent two consecutive monthly injections of anti-VEGF. The mean interval between baseline and final follow-up was $62.4 \pm 24.8$ (range 28 - 147) days.

Table 1

Patient basic characteristics

\begin{tabular}{ll} 
Eyes (no.) & $\mathbf{2 8}$ \\
\hline Eyes (OS/OD) & $11 / 17$ \\
Sex (male/female) & 12 / 16 \\
Diagnosis (CRVO/BRVO) & $15 / 13$ \\
Ages (years) & $64.18 \pm 10.97$ \\
SRF (no.) & 19 / 9 \\
\hline $\begin{array}{l}\text { no.: number; OD: oculus dexter; OS: oculus sinister; RVO: retinal vein occlusion; BRVO: branch retinal } \\
\text { vein occlusion; CRVO: central retinal vein Occlusion; SRF: subretinal fluid. Data are presented as a } \\
\text { number or mean } \pm \text { standard deviation (SD). }\end{array}$ \\
\hline
\end{tabular}

\section{Bcva Improved And Cmt Was Decreased After Anti-vegf Treatment}

The BCVA was improved gradually after anti-VEGF treatments. As shown in Table 2, the mean BCVA (logMAR) increased from $0.83 \pm 0.56$ (baseline) to $0.62 \pm 0.56$ (1st injection, $p>0.05$ ) and $0.49 \pm 0.47$ (2nd injection, $p>0.05$ ). 
Table 2

The changes of visual acuity and other parameters examined with OCTA before and after anti-VEGF treatment

\begin{tabular}{|c|c|c|c|c|}
\hline & Pre & $1 \mathrm{Mo}$ & $2 \mathrm{Mo}$ & $P$ \\
\hline BCVA (logMAR) & $0.83 \pm 0.56$ & $0.62 \pm 0.56$ & $0.49 \pm 0.47$ & $>0.05$ \\
\hline \multicolumn{5}{|l|}{ Density (\%) } \\
\hline SCP & $40.87 \pm 5.60$ & $41.67 \pm 5.93$ & $41.16 \pm 5.69$ & $>0.05$ \\
\hline DCP & $41.10 \pm 6.10$ & $41.83 \pm 5.20$ & $41.53 \pm 6.80$ & $>0.05$ \\
\hline $\mathrm{FAZ}\left(\mathrm{mm}^{2}\right)$ & $0.42 \pm 0.25$ & $0.40 \pm 0.20$ & $0.40 \pm 0.20$ & $>0.05$ \\
\hline CMT $(\mu \mathrm{m})$ & $447.54 \pm 217.93$ & $260.39 \pm 69.32^{\star}$ & $247.00 \pm 61.96^{\star}$ & $<0.05$ \\
\hline $\mathrm{NPA}\left(\mathrm{mm}^{2}\right)$ & $2.58 \pm 2.32$ & $1.64 \pm 1.66$ & $1.35 \pm 1.64^{*}$ & $<0.05$ \\
\hline HRF in outer retina (no.) & $13.71 \pm 5.66$ & $7.93 \pm 3.96^{*}$ & $5.18 \pm 3.30^{*}$ & $<0.05$ \\
\hline Height of SRF ( $\mu \mathrm{m})$ & $30.85 \pm 21.35$ & $11.14 \pm 9.95^{\star}$ & $4.02 \pm 5.47^{*}$ & $<0.05$ \\
\hline \multicolumn{5}{|c|}{$\begin{array}{l}\text { OCTA: Optical coherence tomography angiography; Pre: pre-treatment; Mo: month; BCVA: best- } \\
\text { corrected visual acuity; LogMAR: the logarithm of the minimum angle of resolution; SCP: superficial } \\
\text { capillary plexus; DCP: deep capillary plexus; FAZ: the area of the foveal avascular zone; CMT: central } \\
\text { macular thickness; NPA: nonperfusion area; HRF: hyperreflective foci; no.: number; SRF: subretinal } \\
\text { fluid. Data are shown as mean } \pm \text { SD. * means } p<0.05 \text { when compared with pretreatment. }\end{array}$} \\
\hline
\end{tabular}

CMT was decreased significantly after anti-VEGF treatments (Table 2), i.e., $447.54 \pm 217.93 \mu \mathrm{m}$ (baseline), $260.39 \pm 69.32 \mu \mathrm{m}$ (1st injection), and $247.00 \pm 61.96 \mu \mathrm{m}$ (2nd injection).

\section{Npa And Hrf Were Decreased After Anti-vegf Treatment}

After anti-VEGF treatment, NPA was gradual decreased, i.e., $2.58 \pm 2.32 \mathrm{~mm}^{2}$ (baseline), $1.64 \pm 1.66 \mathrm{~mm}^{2}$ (1st injection), and $1.35 \pm 1.64 \mathrm{~mm}^{2}$ (2nd injection). At the 2nd follow-up, the NPA wad decreased significantly compared to the baseline $(p<0.05$, Table 2$)$.

Analysis of the OCTA parameters revealed that the HRF was distributed across all retinal layers. To study the effect of activated microglia on OBRB, the number of HRF in the outer retinas was counted and analyzed (Fig. 1). The results showed that significant decreased number of HRF in outer retina after antiVEGF treatment, i.e., $13.71 \pm 5.66$ (baseline), $7.93 \pm 3.96$ (1st injection), and $5.18 \pm 3.30$ (2nd injection), respectively $(p<0.05)$.

\section{Srf Was Decreased Significantly After Anti-vegf Treatment}


In this cohort, 19 eyes out of 28 patients were presented with SRF (Table 1). After anti-VEGF treatment, the height of SRF was gradually decreased $(p<0.05)$ or even disappeared (Table 2$)$. In one patient with SRF as shown in Fig. 2, the height of SRF was gradually decreased, i.e., $30.85 \pm 21.35 \mu \mathrm{m}$ (baseline), $11.14 \pm 9.95 \mu \mathrm{m}$ (1st injection), and $4.02 \pm 5.47 \mu \mathrm{m}$ (2nd injection), respectively.

\section{The microstructure of outer retina was partially restored after anti-VEGF injections.}

Besides the absorption of SRF or the resolution of ME, the microstructures of the outer retina, including ellipsoid zone (EZ), interdigitation zone (IZ)/RPE layer, were partially restored, which became more discernible after anti-VEGF treatment (Fig. 3).

\section{No alterations for Microvascular Density and FAZ after anti-VEGF treatment}

Regarding retinal microvascular density in the SCP and DCP analyzed with OCTA, no significant difference was found for the microvascular density in the foveal, parafoveal, and whole macular sectors before and after treatment as shown in Table 2.

The FAZ areas remained unchanged from $0.42 \pm 0.25 \mathrm{~mm}^{2}$ (baseline), $0.40 \pm 0.20 \mathrm{~mm}^{2}$ (1 st injection) to $0.40 \pm 0.20 \mathrm{~mm}^{2}$ (2nd injection), respectively, as shown in Table 2 .

\section{Discussion}

RVO is the second common retinal vascular disease that impairs vision after diabetic retinopathy [7]. Although there are many treatment measures, such as laser photocoagulation, pharmacological agents, and surgical options [1], intravitreal injection of anti-VEGF improved visual acuity and reduce the macular edema, which is becoming the first-line therapy for the treatment of RVO-ME [4]. The research showed that VEGF not only activates vascular endothelial growth factor receptor 2 (VEGFR2), leading to the reduction of tight junctions and the destruction of BRB, but also stimulates endothelial cells to production of a vasodilator, nitric oxide (NO), and increase intercellular cell adhesion molecule-1 (ICAM-1) expression $[13,14]$. Besides VEGF is also an important stimulator for the activation of inflammatory cells, such as microglia and mononuclear phagocytes. Through binding VEGF, anti-VEGF therapy becomes the first-line therapy in the treatment of RVO-ME [15].

In this study, the improvement of visual acuity and decrease of macular edema were observed after antiVEGF treatments, demonstrating that anti-VEGF therapy is very effective for patients with RVO-ME. Besides, BCVA and CMT, other parameters related to RVO were also analyzed, including vessel density, FAZ, HRF, and SRF, etc. The vessel density of SCP and DCP can be well identified and calculated with OCTA auto-segmentation software. Winegarner A. et.al[16] reported that the relationship between the decreased vessel density and visual impairment in RVO. The FAZ area was suggested as a biomarker to for local retinal ischemia within macular region [17, 18], and previous study showed that progressive enlargement of FAZ after anti-VEGF therapy in cystoid ME secondary to diabetic retinopathy or RVO [19], 
indicating the macular ischemia. However, in our study, no significant changes for vessel density and FAZ area before and after anti-VEGF treatments, which is consistent with the previous study [20].

The mechanism of NPA formation remains unclear. Some researchers speculated the causative may compression of capillaries by swollen retinal tissues, arterial insufficiency based on back pressure by the obstructed veins, or capillary occlusion due to leukocyte accumulation at the endothelium. NPA usually considered similar to ischemia, and the size of NPA can distinguish the ischemic and nonischemic types in RVO [4]. Here, the decrease of NPA was observed after anti-VEGF treatment. The present study indicated that early and promptly treatment will benefit the patients more due to alleviate the ischemic condition of macula. It is suggested that anti-VEGF improved blood flow and oxygen supply, reduced the expression of VEGF and ICAM-A, and suppressed the accumulation of leukocyte, leading to reduction of NPA. The detailed mechanisms for NPA decrease after anti-VEGF treatment remains further exploration.

Chen et.al [21] reported that HRF associated with acquired vitelliform lesions were of RPE origin from a donor eye. HRF might as a biomarker associated with the activity and severity of choroideremia. It related to macrophages/microglial activation or progressive PRE degeneration [22]. HRF position was positively associated with final visual outcome, and varied in different diseases depending on various pathologic mechanisms [23]. With aid of OCTA, we found HRF distributed in all the retina layers in patients with RVO$\mathrm{ME}$, and the number of HRF were decreased significantly after anti-VEGF treatment. At the same time, the restoration of microstructure of the outer retina was observed, including the myoid zone (MZ), ellipsoid zone (EZ), the outer segments of the photoreceptors (OS) and interdigitation zone (IZ) / RPE after antiVEGF therapy. It also showed that SRF absorption might contribute to the repair of the microstructure and function of retina. Kang, J. W. et.al [24] reported that the more rupture of the external limiting membrane (ELM) structure, the less intact, and the greater the amount of HRF, no matter CRVO or BRVO.

The CMT, defined as the mean thickness of the central $1 \mathrm{~mm}$ circle centered on the fovea, is the most used parameter to evaluate ME [25]. The significant reduction of CMT in our study implied that ME or SRF were absorbed rapidly after anti-VEGF. RPE functions as to maintain the permeability of transepithelial gradient generated by ion pumps and ion channels, and to avoid paracellular flow. The fluid movement between cells follows the principle of osmotic gradient, flowing from the retina toward to the choroid [6]. In RVO-ME, the function of RPE is impaired, resulting in restricted drainage function, causing SRF. The decrease of SRF after anti-VEGF treatment indicated the improvement of RPE drainage function by antiVEGF, although the detailed mechanisms need further study.

Pervious study showed that the level of inflammatory cytokines, including VEGF, was elevated in either aqueous or vitreous samples, which was associated with the severity of ME $[4,26]$. VEGF has also been reported to increase vascular permeability by increasing the phosphorylation of tight junction proteins. As mentioned before, the mechanism for RVO-ME is complex [27], with other factors involved, such as interleukin 6 (IL-6), monocyte chemoattractant protein-1 (MCP-1), and pigment epithelial-derived factor (PEDF) $[3,7]$. Thus, it would be more accurate if personized treatment for RVO-ME is initiated. More indepth research and more precise combination treatments are needed in the future. 
In summary, anti-VEGF is effective for the treatment of RVO-ME, in terms of visual acuity improve and reduction of macular edema. The absorption of SRF indicated the restoration of RPE drainage function by anti-VEGF treatment; while the decrease of HRF and NPA might indicate anti-inflammatory effect by anti-VEGF treatment to deactivate the inflammatory cells (Fig. 4).

\section{List Of Abbreviations}

RPE: Retinal pigment epithelium; RVO-ME: Retinal vein occlusion; Anti-VEGF: Anti-vascular endothelial growth factor ; OCTA: Optical coherence tomography angiography; BCVA: Best-corrected visual acuity; FAZ: Foveal avascular zone; NPAs: Nonperfusion areas; CMT: Central macular thickness; SCP: Vascular density of the superficial capillary plexus; DCP: Vascular density of the deep capillary plexus; SRF:

Subretinal fluid; HRF: Hyperreflective foci; CRVO: Central retinal vein occlusion; BRVO: Branch retinal vein occlusion; HRVO: Hemiretinal vein occlusion; ME: Macular edema; BRB: Blood-retinal barrier; IOP: Intraocular pressure; IZ: Interdigitation zone; EZ: Ellipsoid zone; MZ: Myoid zone; ELM: External limiting membrane; OD: Oculus dexter; OS: Oculus sinister; LogMAR: The logarithm of the minimum angle of resolution

\section{Declarations}

\section{Ethics approval and consent to participate}

This study was approved by the Clinical Research Ethical Committee of Shanghai General Hospital affiliated to Shanghai Jiao Tong University (Permits No. 2020KY205) and adhered to the principles of the Declaration of Helsinki. All individual participants provided written informed consent.

\section{Consent for publication}

Written informed consents were obtained from all participants for the publication of clinical information and images.

\section{Availability of data and materials}

All data generated or analyzed during this study are included in this published article and its supplementary information files.

\section{Competing interests}

The authors declare that they have no competing interests.

\section{Funding}

This study was supported by the National Natural Science Foundation of China (81970810, 81970811, 82171062); the Science and Technology Commission of Shanghai Municipality (18411953400, 
19495800700); Domestic Science and Technology Cooperation Project of Shanghai Municipal Science and Technology Commission (21015800700); National Key Basic Research Program of China (2017YFA0104100); and National Major Scientific and Technological Special Project for "Significant New Drugs Development" during the Thirtieth Five-year Plan Period (2019ZX09301113).

\section{Authors' contributions}

Qian Yang: Data collection; Analysis and interpretation of data; Writing the manuscript; Statistical expertise

Dawei Luo and Qinghua Qiu: Provision of clinical sample data; Obtaining funding Guo-Tong Xu: Conception and design; Administrative, technical, or material support; Supervision Jingfa Zhang: Conception and design; Administrative, technical, or material support; Critical revision of the manuscript; Obtaining funding; Supervision

All authors read and approved the final manuscript.

\section{Acknowledgements}

Not applicable.

\section{References}

1. Zeng $Y$, Yang K, Wang F, Zhou L, Hu Y, Tang M, et al. The glucagon like peptide 1 analogue, exendin-4, attenuates oxidative stress-induced retinal cell death in early diabetic rats through promoting Sirt1 and Sirt3 expression. Experimental eye research 2016,151:203-11

2. Scott IU, Campochiaro PA, Newman NJ, Biousse V. Retinal vascular occlusions. Lancet (London, England) 2020,396(10266):1927-40

3. Ascaso FJ, Huerva V, Grzybowski A. The role of inflammation in the pathogenesis of macular edema secondary to retinal vascular diseases. Mediators of inflammation 2014,2014:432685

4. Hirano Y, Suzuki N, Tomiyasu T, Kurobe R, Yasuda Y, Esaki Y, et al. Multimodal Imaging of Microvascular Abnormalities in Retinal Vein Occlusion. Journal of clinical medicine 2021,10(3)

5. Daruich A, Matet A, Moulin A, Kowalczuk L, Nicolas M, Sellam A, et al. Mechanisms of macular edema: Beyond the surface. Progress in retinal and eye research 2018,63:20-68

6. Willermain F, Libert S, Motulsky E, Salik D, Caspers L, Perret J, et al. Origins and consequences of hyperosmolar stress in retinal pigmented epithelial cells. Frontiers in physiology 2014,5:199

7. Cehofski LJ, Honoré B, Vorum H. A Review: Proteomics in Retinal Artery Occlusion, Retinal Vein Occlusion, Diabetic Retinopathy and Acquired Macular Disorders. International journal of molecular sciences 2017,18(5) 
8. Kaur C, Foulds WS, Ling EA. Blood-retinal barrier in hypoxic ischaemic conditions: basic concepts, clinical features and management. Progress in retinal and eye research 2008,27(6):622-47

9. Kashani AH, Chen CL, Gahm JK, Zheng F, Richter GM, Rosenfeld PJ, et al. Optical coherence tomography angiography: A comprehensive review of current methods and clinical applications. Progress in retinal and eye research 2017,60:66-100

10. Bolz M, Schmidt-Erfurth U, Deak G, Mylonas G, Kriechbaum K, Scholda C. Optical coherence tomographic hyperreflective foci: a morphologic sign of lipid extravasation in diabetic macular edema. Ophthalmology 2009,116(5):914-20

11. Hasegawa T, Masuda N, Ogata N. Highly reflective line in optical coherence tomography images of eyes with macular edema associated with branch retinal vein occlusion. American journal of ophthalmology 2015,159(5):925-33.e1

12. Wu J, Zhang C, Yang Q, Xie H, Zhang J, Qiu Q, et al. Imaging Hyperreflective Foci as an Inflammatory Biomarker after Anti-VEGF Treatment in Neovascular Age-Related Macular Degeneration Patients with Optical Coherence Tomography Angiography. BioMed research international 2021,2021:6648191

13. Noma H, Funatsu H, Mimura T, Eguchi S, Hori S. Soluble vascular endothelial growth factor receptor2 and inflammatory factors in macular edema with branch retinal vein occlusion. American journal of ophthalmology 2011,152(4):669-77.e1

14. Roberts WG, Palade GE. Increased microvascular permeability and endothelial fenestration induced by vascular endothelial growth factor. Journal of cell science 1995,108 (Pt 6):2369-79

15. Sangroongruangsri S, Ratanapakorn T, Wu O, Anothaisintawee T, Chaikledkaew U. Comparative efficacy of bevacizumab, ranibizumab, and aflibercept for treatment of macular edema secondary to retinal vein occlusion: a systematic review and network meta-analysis. Expert review of clinical pharmacology 2018,11(9):903-16

16. Winegarner A, Wakabayashi T, Fukushima Y, Sato T, Hara-Ueno C, Busch C, et al. Changes in Retinal Microvasculature and Visual Acuity After Antivascular Endothelial Growth Factor Therapy in Retinal Vein Occlusion. Investigative ophthalmology \& visual science 2018,59(7):2708-16

17. Adhi M, Filho MA, Louzada RN, Kuehlewein L, de Carlo TE, Baumal CR, et al. Retinal Capillary Network and Foveal Avascular Zone in Eyes with Vein Occlusion and Fellow Eyes Analyzed With Optical Coherence Tomography Angiography. Investigative ophthalmology \& visual science 2016,57(9):Oct486-94

18. Balaratnasingam C, Inoue M, Ahn S, McCann J, Dhrami-Gavazi E, Yannuzzi LA, et al. Visual Acuity Is Correlated with the Area of the Foveal Avascular Zone in Diabetic Retinopathy and Retinal Vein Occlusion. Ophthalmology 2016,123(11):2352-67

19. Feucht N, Schönbach EM, Lanzl I, Kotliar K, Lohmann CP, Maier M. Changes in the foveal microstructure after intravitreal bevacizumab application in patients with retinal vascular disease. Clinical ophthalmology (Auckland, NZ) 2013,7:173-8 
20. Ghasemi Falavarjani K, lafe NA, Hubschman JP, Tsui I, Sadda SR, Sarraf D. Optical Coherence Tomography Angiography Analysis of the Foveal Avascular Zone and Macular Vessel Density After Anti-VEGF Therapy in Eyes With Diabetic Macular Edema and Retinal Vein Occlusion. Investigative ophthalmology \& visual science 2017,58(1):30-4

21. Chen KC, Jung JJ, Curcio CA, Balaratnasingam C, Gallego-Pinazo R, Dolz-Marco R, et al. Intraretinal Hyperreflective Foci in Acquired Vitelliform Lesions of the Macula: Clinical and Histologic Study. American journal of ophthalmology 2016,164:89-98

22. Romano F, Arrigo A, MacLaren RE, Charbel Issa P, Birtel J, Bandello F, et al. HYPERREFLECTIVE FOCI AS A PATHOGENETIC BIOMARKER IN CHOROIDEREMIA. Retina (Philadelphia, Pa) 2020,40(8):163440

23. Mo B, Zhou HY, Jiao X, Zhang F. Evaluation of hyperreflective foci as a prognostic factor of visual outcome in retinal vein occlusion. International journal of ophthalmology 2017,10(4):605-12

24. Kang JW, Lee H, Chung H, Kim HC. Correlation between optical coherence tomographic hyperreflective foci and visual outcomes after intravitreal bevacizumab for macular edema in branch retinal vein occlusion. Graefe's archive for clinical and experimental ophthalmology = Albrecht von Graefes Archiv fur klinische und experimentelle Ophthalmologie 2014,252(9):1413-21

25. Brown DM, Campochiaro PA, Singh RP, Li Z, Gray S, Saroj N, et al. Ranibizumab for macular edema following central retinal vein occlusion: six-month primary end point results of a phase III study. Ophthalmology 2010,117(6):1124-33.e1

26. Campochiaro PA, Akhlaq A. Sustained suppression of VEGF for treatment of retinal/choroidal vascular diseases. Progress in retinal and eye research 2020:100921

27. Campochiaro PA, Heier JS, Feiner L, Gray S, Saroj N, Rundle AC, et al. Ranibizumab for macular edema following branch retinal vein occlusion: six-month primary end point results of a phase III study. Ophthalmology 2010,117(6):1102-12.e1

\section{Figures}

\section{Pre}

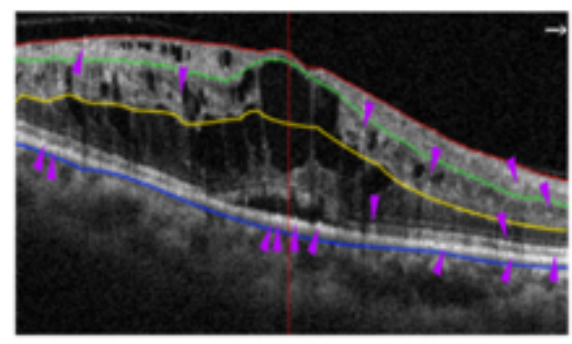

$1 \mathrm{Mo}$

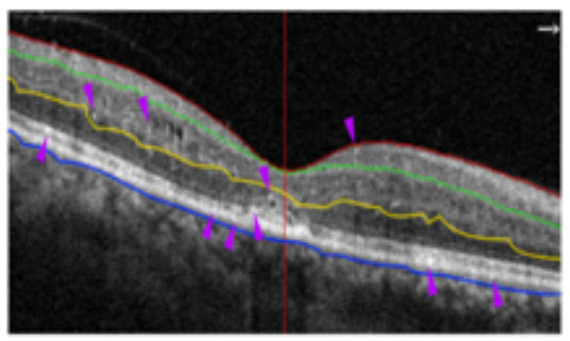

$2 \mathrm{Mo}$

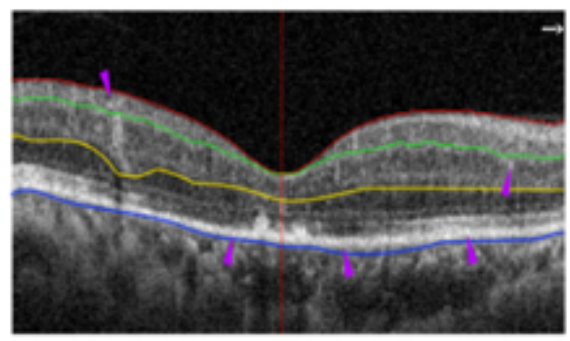

\section{Figure 1}


The changes of hyperreflective foci (HRF) in 66-years-old female patient with CRVO-ME before and after anti-VEGF treatments. The representative OCTA images at the $6 \times 6 \mathrm{~mm}^{2}$ sections centered on the fovea showed the gradual decrease of the number of HRF (purple triangle arrow) after anti-VEGF treatment. HRF were distributed throughout the whole retina before treatment. HRF in the outer retina (beneath the yellow line and above the blue line) was decreased significantly after treatment. The macular edema was also decreased after 2 consecutive anti-VEGF treatments.

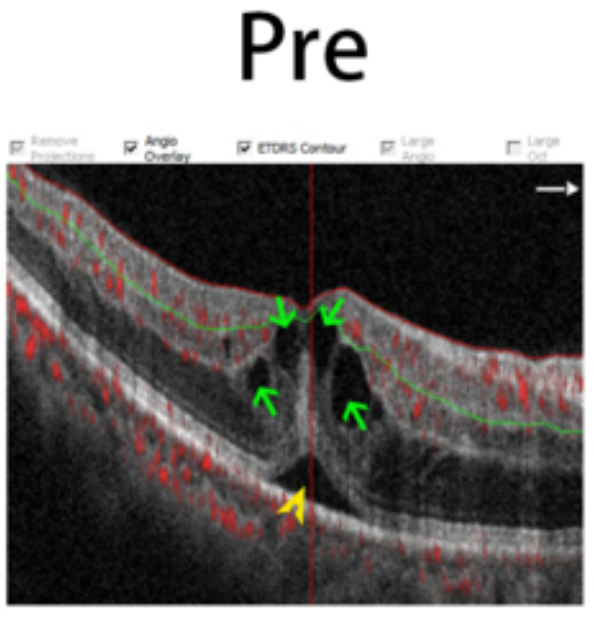

\section{Mo}

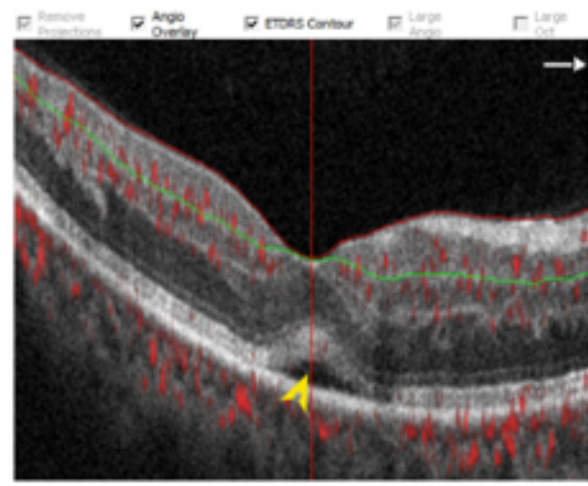

$2 \mathrm{Mo}$

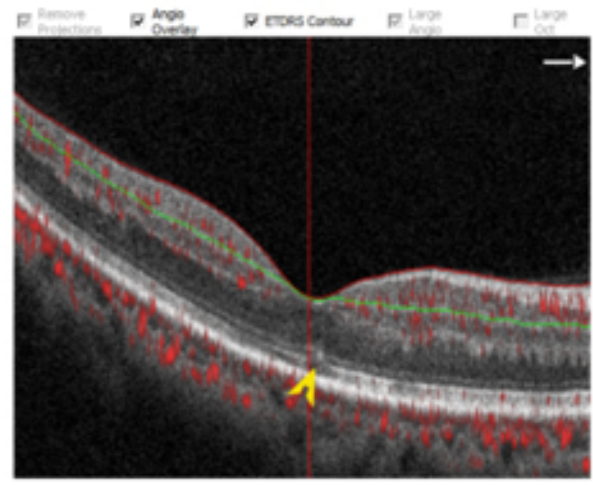

Figure 2

The changes of subretinal fluid (SRF) in 54-years-old female patient with BRVO-ME before and after antiVEGF treatments. The representative OCTA images at the $6 \times 6 \mathrm{~mm}^{2}$ sections centered on the fovea showed the resolution of SRF (yellow arrow head) and macular edema (ME, green arrow) before and after intravitreal injection of anti-VEGF treatments. The SRF was completely absorbed after 2 consecutive antiVEGF treatments.

Pre

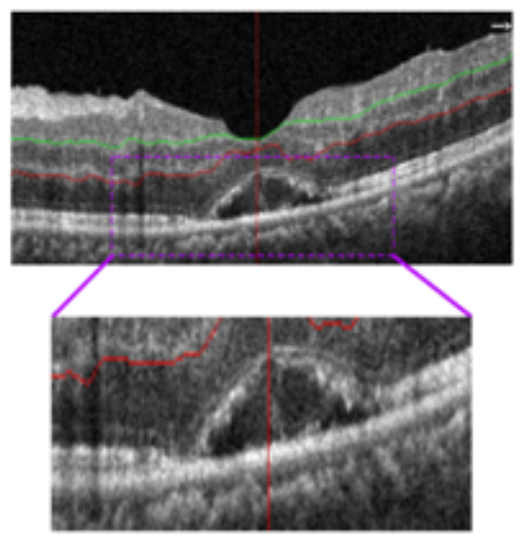

$1 \mathrm{Mo}$

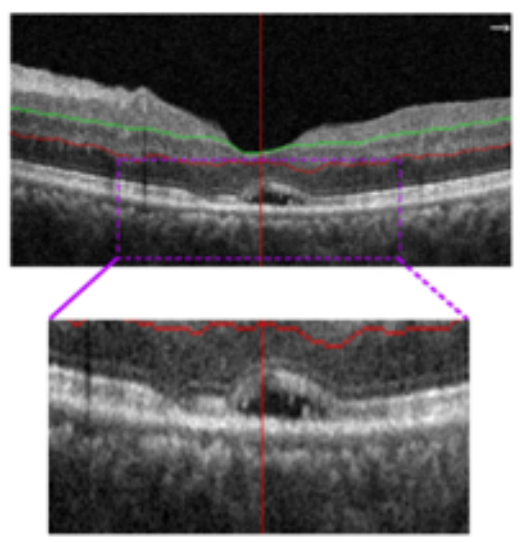

$2 \mathrm{Mo}$

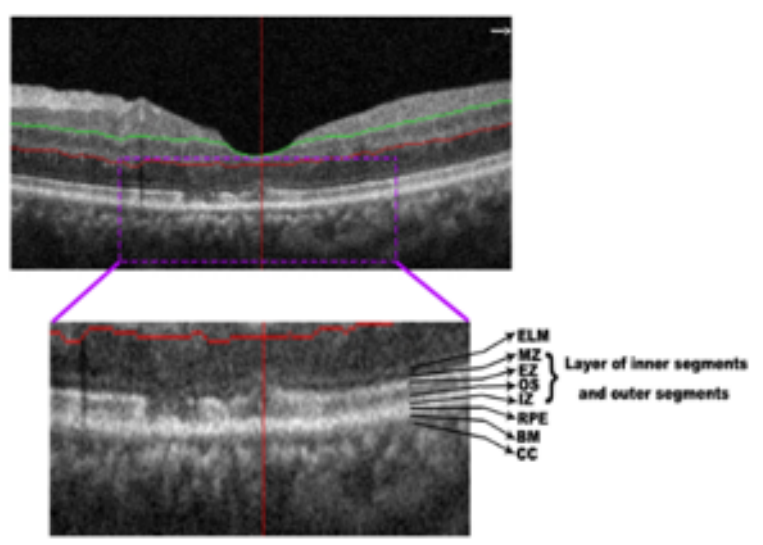




\section{Figure 3}

The morphological changes of outer retinal in 61-years-old female patient with BRVO-ME before and after anti-VEGF treatment. The representative OCTA images at the $6 \times 6 \mathrm{~mm}^{2}$ sections centered on the fovea showed the microstructures of , including the myoid zone (MZ), ellipsoid zone (EZ), the outer segments of the photoreceptors (OS) and interdigitation zone (IZ) / retinal pigment epithelial (RPE) layer, were partially restored, while the height of SRF and macular edema was decreased after 2 consecutive anti-VEGF treatments. ELM: External limiting membrane; BM: Bruch's membrane; CC: Choriocapillaris
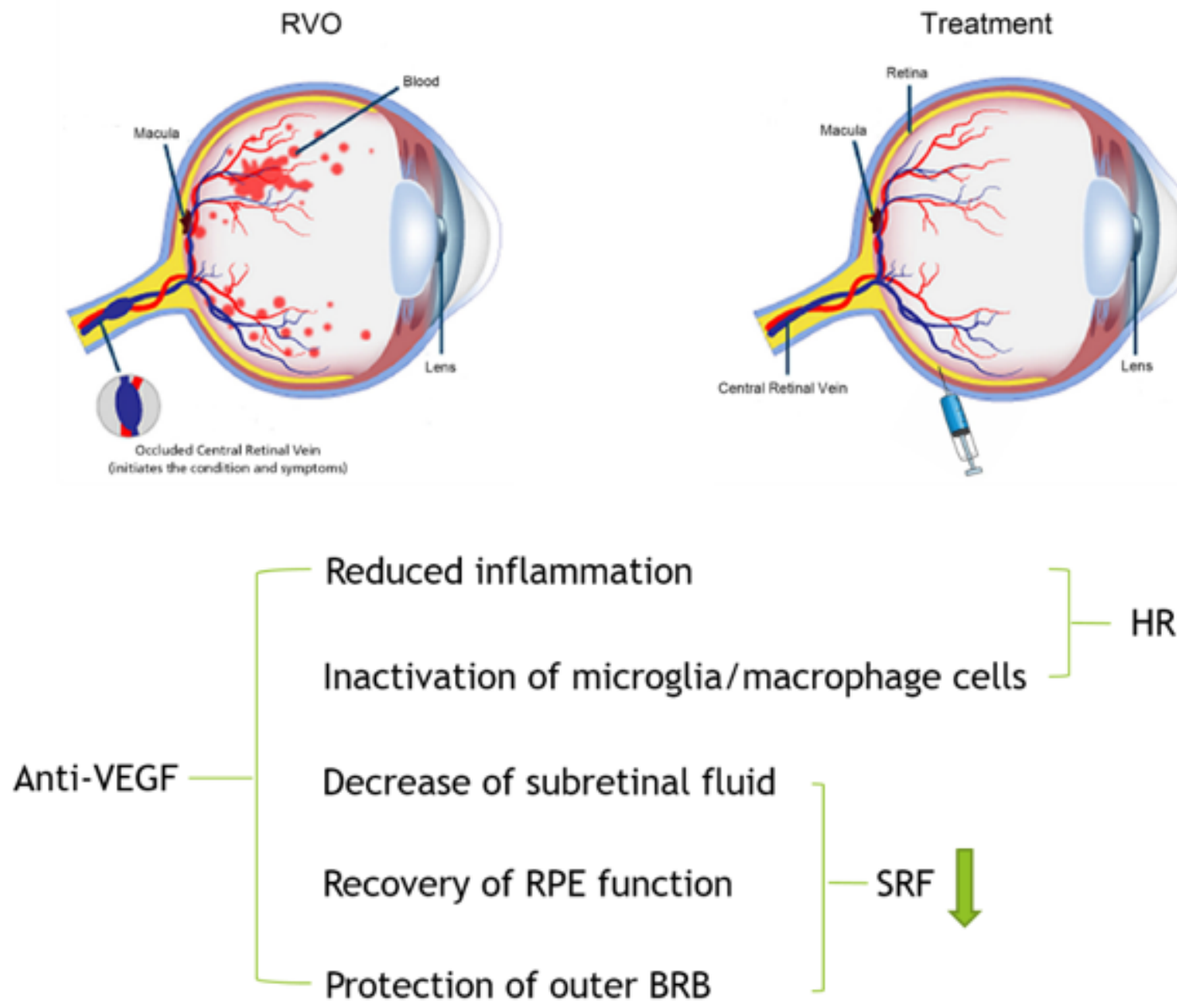

Figure 4

Diagram of the possible mechanism of anti-VEGF treatment for RVO-ME. On the one hand, anti-VEGF can reduce the number of HRF by inactivating microglia/macrophages cells. On the other hand, by restoring the drainage function of RPE and repairing the oBRB barrier to reduce the accumulation of SRF, and finally achieving the treatment of RVO-ME. 